\title{
A mouse model of gestation-specific transient hyperglycemia for translational studies
}

\author{
H Y Li1 , Y X Liu'1,2, L Harvey³, S Shafaeizadeh ${ }^{3}$, E M van der Beek ${ }^{3,4}$ and W Han 1,5,6 \\ ${ }^{1}$ Laboratory of Metabolic Medicine, Singapore Bioimaging Consortium, A*STAR, Singapore, Republic of Singapore \\ 2Danone Nutricia Research, Singapore, Republic of Singapore \\ ${ }^{3}$ Danone Nutricia Research, Utrecht, The Netherlands \\ 4Department of Pediatrics, University Medical Centre Groningen, Groningen, The Netherlands \\ Institute of Molecular and Cell Biology, A*STAR, Singapore, Republic of Singapore \\ ${ }^{6}$ School of Laboratory Medicine and Life Science, Wenzhou Medical University, Wenzhou, Zhejiang, China
}

Correspondence should be addressed to W Han: wh10@cornell.edu

\begin{abstract}
The prevalence of gestational diabetes mellitus (GDM) is estimated at $14 \%$ globally, and in some countries, such as Singapore, exceeds $20 \%$. Both women and children exposed to GDM have an increased risk of later metabolic diseases, cardiovascular disease and other health issues. Beyond lifestyle changes and pharmaceutical intervention using existing type 2 diabetes medications for expecting women, there are limited treatment options for women with GDM; targeting better outcomes of potentially affected infants is unexplored. Numerous animal models have been generated for understanding of pathological processes of GDM development and for development of treatment strategies. These models, however, suffer from limited windows of opportunity to examine risk factors and potential intervention options. By combining short-term high-fat diet (HFD) feeding and low-dose streptozotocin (STZ) treatments before pregnancy, we have established a mouse model with marked transient gestation-specific hyperglycemia, which allows testing of nutritional and pharmacological interventions before, during and beyond pregnancy.
\end{abstract}

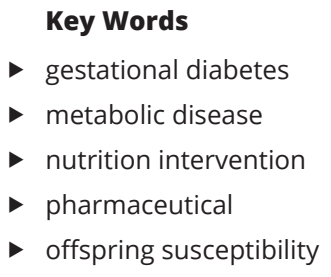

Journal of Endocrinology (2020) 244, 501-510

\section{Introduction}

Gestational diabetes mellitus (GDM) is a serious condition that affects maternal and child health but may still be relatively neglected in current practice. It is currently estimated to affect $14 \%$ of pregnancies worldwide (Balsells et al. 2009). In high-risk populations, such as in Singapore, over 20\% pregnancies are diagnosed with GDM (Chong et al. 2014, Yew et al. 2014). The rate is even higher in subsequent pregnancies without preventative measures (Getahun et al. 2010, Ehrlich et al. 2011) and prevalence continues to climb as known risk factors, such as maternal age and maternal overweight/obesity, accumulate over time. GDM, defined as glucose intolerance that develops and is first diagnosed during pregnancy, significantly increases the risk of adverse consequences for both mothers and offspring (Kampmann et al. 2015, Farahvar et al. 2019). There is an urgent need to develop strategies to prevent and treat these short- and long-term sequelae.

The underlying pathogenic mechanisms of GDM and their contribution to adverse outcomes for mother and offspring remain to be fully elucidated. It is recognized that the disease shares the same multifactorial causes of type 2 diabetes (T2D), namely insulin resistance and pancreatic $\beta$ cell dysfunction, which may have been pre-existing or induced by pregnancy (Kaaja \& Ronnemaa 2008). Many women with GDM seem to have $\beta$ cell dysfunction that develops on a background of chronic insulin 
resistance that is often present already prior to pregnancy. Elevated placental hormone levels during pregnancy further exacerbate insulin resistance with concomitant increases in insulin production; the subsequent failure of adequate $\beta$ cell function compensation results in GDM. For most women, the symptoms of GDM usually resolve shortly after delivery, however, the risk of developing T2D within 10 years of the GDM pregnancy is high (Tutino et al. 2014).

The first-line treatment of GDM is lifestyle intervention, including a calorie-controlled diet plan and physical exercise (Yamamoto et al. 2018). For uncontrolled hyperglycemia that persists despite lifestyle modifications, diabetic medications (mainly glyburide, metformin, and eventually insulin) are usually prescribed (Bimson et al. 2017, Farrar et al. 2017). The general principle for the prescription of these medications is similar to the management of T2D; these treatments may effectively manage hyperglycemia during pregnancy, but long-term effects on maternal health as well as consequences for the offspring have rarely been studied (Balsells et al. 2009). The lack of knowledge on the long-term outcome of these management strategies is concerning (Logan et al. 2017). Except for an increased risk of neonatal hypoglycemia, GDM offspring are considered healthy and thus taken care of in general maternity wards (except in cases of premature birth). Risk assessments or preventative treatment strategies to manage the consequences of GDM exposure for the offspring are lacking and potential adverse pregnancy exposures are often not taken into account during post-natal growth and health assessments of offspring of women with GDM, despite the reported differences in adiposity outcomes (Logan et al. 2017). Current diagnostic criteria acknowledge the acute needs regarding glycemia management but ignore the linear relationship between maternal glycemia (and medication use) and infant outcomes (Balsells et al. 2009, Lowe et al. 2019). There is an urgent need to develop efficacious therapeutic options and guidelines targeting specific patient subpopulations as well as offspring development.

Animal models of GDM have been generated to better understand the etiology of the disease and its pathophysiological impact on both mothers and, sometimes, their offspring (Pasek \& Gannon 2013). Modeling approaches include surgical manipulation (e.g., $\beta$ cell removal), pharmacological treatments (e.g., streptozotocin induced $\beta$ cell loss), nutritional manipulations (e.g., high fat or high fat/high sugar (obesogenic) diet), and genetic modifications (e.g., leptin receptor knockout), mostly in mice and rats (well-reviewed in Pasek \& Gannon 2013). While contributing to our knowledge on certain aspects of GDM, each model so far is limited in its ability to capture the heterogeneous pathogenesis and, particularly, the transient characteristics of human GDM, thus offering limited opportunities to conduct intervention studies for the development of better treatments. Continued innovation in the development of animal models of GDM with improved translational value (i.e. a model that is truly transient) is needed.

Our aim was to develop a GDM mouse model optimized for future development of preventative and therapeutic interventions for mothers and infants. We utilized a twohit approach including a short-term high fat diet (HFD) feeding to induce pre-pregnancy insulin resistance, and multiple low-dose streptozotocin (STZ) injections prior to pregnancy to disrupt $\beta$ cell compensation in mice.

\section{Materials and methods}

\section{Animal welfare}

All experiments involving animals were reviewed and approved by the Institutional Animal Care and Use Committee (IACUC) of the Agency for Science Technology and Research (A*STAR), adhering to the National Advisory Committee on Laboratory Animal Research guidelines. Experiments were performed in an Assessment and Accreditation of Laboratory Animal Care accredited specific pathogen free (SPF) facility located in Biopolis, Singapore.

\section{Experimental design}

The clinical diagnostic marker for GDM is glucose handling. We devised a two-hit approach to induce hyperglycemia during pregnancy. The first hit was to induce insulin resistance; this could be reliably produced, without overt obesity, by short-term feeding with HFD (Winzell \& Ahren 2004, Shoelson et al. 2007, Heydemann 2016). The second hit was to prevent $\beta$ cell compensation from overcoming insulin resistance. High-dose STZ effectively kills $\beta$ cells, rendering a type 1 diabetic phenotype (Wu \& Huan 2008), while multiple low-dose STZ leads to mild hyperglycemia, similar to a mild T2D phenotype (Eleazu et al. 2013). We aimed to control the extent of insulin resistance and $\beta$ cell dysfunction so that (a) the mice would not develop hyperglycemia prior to pregnancy and (b) ideally, they would recover from hyperglycemia after delivery. We used periodic measurements of random blood glucose 
(RBG) levels to monitor development of hyperglycemia and oral glucose tolerance tests (OGTTs) to selectively validate RBG measurements. We chose to use RBG levels over fasting blood glucose levels so as to minimize disturbance to the dams during gestation.

We aimed to find a balance in the experimental manipulations that ideally featured a minimal period of HFD feeding, while maintaining optimal fertility and pregnancy rate. We tested HFD feeding periods of between 4 and 8 weeks combined with various low doses of STZ (either single or multiple injections). After several rounds of small-scale trials ( $n \leq 6$ per group) using combinations of HFD types (45 and 60\%) and duration (up to 2 months),

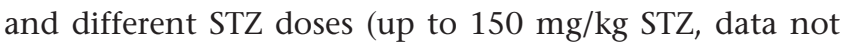
shown), we were able to define a protocol that produced gestation-specific hyperglycemia in a large number of animals that were treated. Briefly, 8-week-old female mice were kept on $60 \%$ HFD for 4 weeks, followed by $60 \mathrm{mg} / \mathrm{kg}$ body weight of STZ once per day for 3 consecutive days. HFD exposure was maintained and mice were monitored for 2 weeks, and then mated and monitored during gestation.

\section{Animal use}

Seven to eight-week-old nulliparous female and male C57Bl/6N mice were purchased in batches from InVivos Pte Ltd. Female mice, bodyweight 16-21 g, were fed ad libitum with 60\% semi-synthetic high fat diet (HFD, D12492, Research Diets) or 10\% fat semi-synthetic control diet (low-fat diet (LFD), D12450J, Research Diets) after 1 week of habituation. Male mice were placed on normal chow diet (1324, Altromin). All diets were irradiated. Male and female mice were housed five per cage before mating and individually after mating, in ventilated cages lined with corncob bedding. All mice were subjected to a $12 \mathrm{~h}$ light-dark cycle (lights on 07:00 h, lights off 19:00 h). All fasting was performed in the animal's home cage: fasting prior to STZ injection was performed for $6 \mathrm{~h}$ during the light phase; fasting for OGTT was performed during the dark phase and first $4 \mathrm{~h}$ of light phase; fasting for tissue harvesting was performed for $4-6 \mathrm{~h}$ during the light phase. Body weight was monitored weekly.

After 1 week of habituation, experimental female mice were assigned to four body weight-matched treatment groups: vehicle injection control on LFD (LFD-Veh), vehicle injection control on HFD (HFD-Veh), STZ treated on LFD (LFD-STZ) and STZ treated on HFD (HFD-STZ). All mice within a single cage received the same treatment. After 4 weeks on LFD or HFD, 6-h fasted mice were treated with either $60 \mathrm{mg} / \mathrm{kg}$ STZ (S0130, Sigma-Aldrich) or vehicle via intraperitoneal injection for 3 consecutive days. STZ was dissolved in $0.1 \mathrm{M}$ sodium citrate buffered to $\mathrm{pH} 4.5$ with citric acid (PHR1416 and 251275, Sigma-Aldrich) and was administered within 15 min of solution preparation. Proven male breeders were pre-trained and selected in-house with a separate cohort of female mice, in parallel to the experimental females. Experimental female mice were housed 1:1 with male breeders for $48 \mathrm{~h}$. Pregnancy was determined via visual inspection at E14. Non-pregnant mice, confirmed by lack of significant weight change, were then mated a second time with a different male breeder. If a female mouse was not pregnant after two rounds of mating, this mouse was retired from the experiment, in order to avoid prolonged HFD exposure. After mating, female mice were individually housed and fed ad libitum with 60\% HFD or LFD according to their experimental group; all were changed to LFD on postnatal day 2 (P2). At P2, litter size was standardized to four to six pups per litter, with at least one female littermate. Male offspring were weaned at P21 to normal chow diet and housed in groups of two. At 8 weeks, subsets of offspring (LFD-Veh and GDM offspring) were fed HFD for an additional 8 weeks, until they were 16 weeks of age.

In order to investigate the outcome of repeated pregnancy on glucose status, breeders $(n=47)$ from selective experimental groups were maintained on grainbased chow diet and subjected to a second round of mating 5 weeks after weaning of their first litter. Breeding protocol was as described earlier, and the same batch of proven male breeders was used.

\section{Blood glucose measurement and oral glucose tolerance test}

Procedures were performed as previously described ( $\mathrm{Li}$ et al. 2014). Blood was sampled from the tail. Blood glucose was measured using a handheld glucose meter (Accu-Chek Performa Nano, Roche). For the monitoring of glycemic changes during pregnancy, random blood glucose (RBG) was sampled between 09:00 and 11:00 h. Random blood glucose indicates that there was no control over the fed status of the animals.

RBG was sampled from dams prior to STZ/Veh injection (on the day of STZ/Veh injection), 1 week after STZ/Veh injection, at embryonic day 15 of pregnancy (E15) and at weaning. OGTT was performed in the dams at E16 as well post weaning. Fasting blood glucose represents the first time point of the OGTT. 
An OGTT was used to assess glucose tolerance of the dams at E16 of each pregnancy and of the adult offspring (of the first pregnancy) within 1 week of P98. A fasting blood glucose measurement was taken prior to administration of $20 \%$ glucose solution ( $2 \mathrm{~g} / \mathrm{kg}$ body weight) via oral gavage. Post-prandial blood glucose levels were determined at 15, 30, 60, 90, 120 min after the oral gavage from a tail blood sample.

\section{Body composition analysis}

Fortnightly body composition analysis was performed to determine the impact of maternal glycemia on the fat mass of the offspring. These procedures were performed as previously described (Gustavsson et al. 2009). Body fat was determined by an MRI analyzer (EchoMRI-100H, EchoMRI) per manufacturer's instructions.

\section{Data analysis and statistics}

Data are presented as mean \pm s.E.M. We compared random blood glucose measurements and plasma insulin levels at E16 and post-partum across treatment and sub-groups using one-way ANOVA, paired with Bonferroni correction multiple comparison post hoc analysis. To assess the change of random blood glucose levels and body weight over time, a mixed-model ANOVA was performed. Body composition and OGTT results were analyzed using a mixed model ANOVA. A value of $P<0.05$ was considered statistically significant.

\section{Results}

Following small scale trials, we tested our experimental design (Fig. 1A) in separate large cohorts to determine success rates and feasibility of our approach. As such, in addition to the HFD-STZ group using the above protocol, we also included three control groups: HFD-Veh, LFD-STZ and LFD-Veh groups. A total of 90 pregnant dams, across all groups, from three independent batches over a period of $\sim 18$ months, were collected and reported here. Both the HFD-STZ and HFD-Veh groups showed moderate body weight increase ( $12 \%)$ after 1 month of HFD. HFD females injected with STZ showed flattened weight gain 2 weeks post injection and resulted in mildly higher body weight ( $~ 8 \%$, comparing to $\sim 28 \%$ for HFD-Veh) compared to the LFD-Veh group prior to mating, and almost identical weight throughout the rest of the experiment (Fig. 1B). Considering the overall mild changes in body weight as well as the potential effects of the stress of the EchoMRI procedure during pregnancy, we did not measure body fat composition prior to and during gestation.

The overall pregnancy ratio was comparable across groups $(\mathrm{LFD}-\mathrm{Veh}=60 \%$, LFD-STZ $=86 \%$, HFD-Veh $=63 \%$, HFD-STZ $=79 \%$ ), showing that the experimental manipulations did not affect pregnancy rate. Total pregnancy success rate was up to $72 \%$, which was high for a model featuring prolonged exposure to HFD.

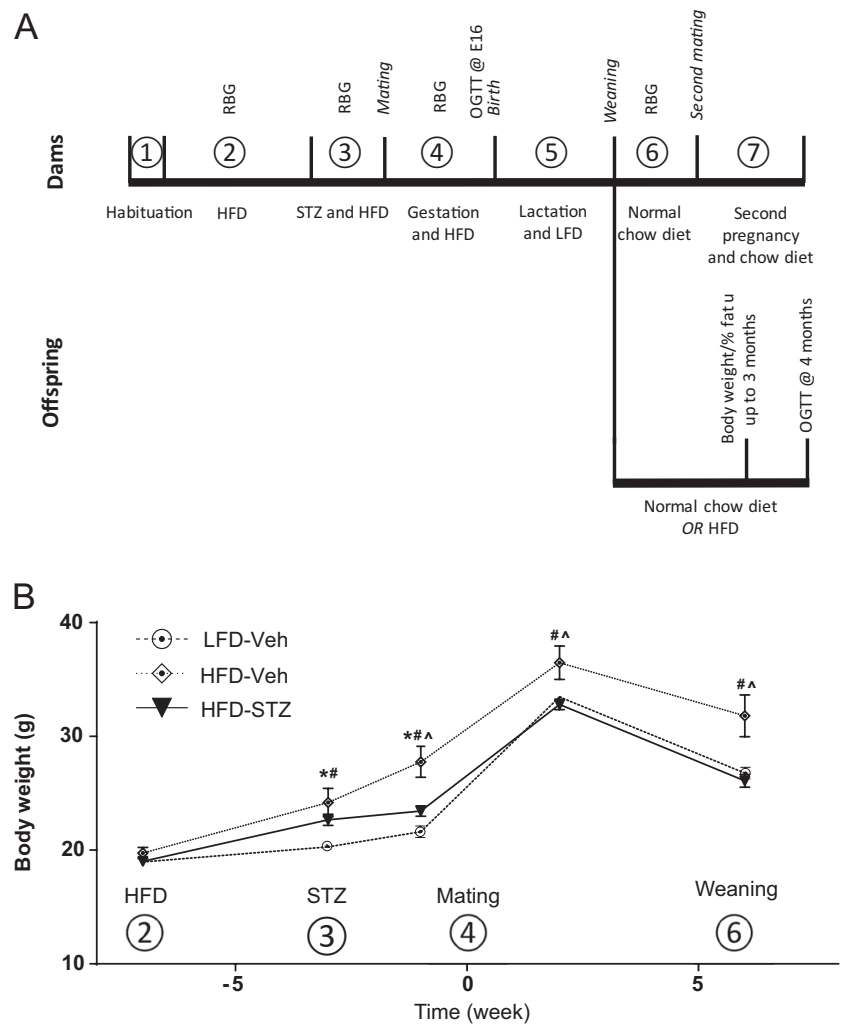

Figure 1

(A) Schematic illustration of the experimental design. Dams: 1. Female mice habituated to the facility for 1 week after arrival. 2. Female mice were fed high-fat diet (HFD) for 4 weeks. 3. Three consecutive low-dose injections of streptozotocin (60 mg/kg, STZ) were administered whilst animals were on HFD. 4. Timed mating and gestation while on HFD, switching to a low-fat diet (LFD) after fasting for oral glucose tolerance test (OGTT). 5. Birth and lactation on LFD. 6. Offspring were weaned and dams were switched to normal chow diet. 7. Dams were mated again 2 weeks post weaning for a second pregnancy. Random blood glucose (RBG) and oral glucose tolerance test (OGTT) were measured at the time points depicted. Offspring: Offspring were weaned and maintained on normal chow diet or HFD from six weeks onwards. Body weight and \% body fat were measured weekly during development for those offspring. Oral glucose tolerance test (OGTT) was conducted in offspring on normal chow diet and HFD at 4 months of age. Numbers in circles correspond to numbers in circles in other figures. (B) Effect of HFD and STZ on body weight gain. The body weight changes of a batch of dams from LFD-Veh $(n=9)$, HFD-Veh $(n=12)$, HFD-STZ $(n=32)$ groups were followed from the beginning till weaning. Statistical significance $P<0.05$ *HFD-STZ vs LFD-Veh; \#HFD-Veh vs LFD-Veh; ^^for HFD-STZ vs HFD-Veh. 
Hereafter, only data from dams which went through gestation are reported, unless specified. Based on the preliminary findings, the HFD-STZ dams $(n=52)$ could be further classified into four subgroups based on random blood glucose at different time points: (1) diabetes prior to gestation (T1D-like, $n=7$ ); (2) isolated impaired glucose tolerance (isoIGT, $n=15$ ); (3) GDM with resolved hyperglycemia after delivery (GDM, $n=24)$; and (4) GDM with persistent diabetes after delivery (T2D-like, $n=6$ ).

For the first gestation: the T1D-like subgroup comprised $\sim 13 \%$ of total HFD-STZ group and developed hyperglycemia (RBG $>12 \mathrm{mM}$ ) before mating. The isoIGT subgroup ( 29\%) remained normoglycemic (RBG $<12 \mathrm{mM})$ throughout the experiment but displayed glucose intolerance during the OGTT. The GDM subgroup $(\sim 46 \%)$ remained normoglycemia for weeks before the first mating, developed hyperglycemia after mid-gestation (RBG $>12 \mathrm{mM}$ by E15), and returned to normoglycemia by weaning. The T2D-like subgroup $(\sim 12 \%)$ initially developed GDM but did not recover to normoglycemia after delivery. These results are summarized in Table 1 . Importantly, those HFD-STZ mice with normoglycemia at time of mating that did not get pregnant (but remained on HFD) remained normoglycemic until the end of the experiment, clearly indicating that pregnancy provides the necessary additional metabolic stress to induce GDM in our model.

\section{GDM model with transient gestation-specific hyperglycemia}

The definition of GDM in rodents was not well established. Given our experimental need to frequently assess glucose status, we used RBG measurements to determine the mouse GDM status. We also tested a 2-h fasting method for glucose assessment, however, found that this was not as reliable as RBG measurement in pregnant mice (Fig. 2A). At E15, pregnant mice from separate trials gave varied and statistically elevated blood glucose readings after 2-h fasting, which could be the result of heightened stress during gestation.

At E15, animals in the HFD-Veh group had almost identical RBG levels $(7.2 \pm 0.2 \mathrm{mM}, n=15)$ as the LFD-Veh control group $(7.1 \pm 0.1 \mathrm{mM}, n=14)$ (Fig. 2B). The LFD-STZ group displayed mildly elevated RBG $(8.7 \pm 0.6 \mathrm{mM}$, $n=9)$ when compared with LFD-Veh group. The isoIGT subgroup had significantly elevated RBG $(9.6 \pm 0.3 \mathrm{mM}$, $n=15, P=0.02$, two-way ANOVA). The GDM subgroup showed severe hyperglycemia $(17.4 \pm 1.1 \mathrm{mM}, n=24$, $P<0.0001$, two-way ANOVA), but this completely resolved by the time of weaning. T1D-like and T2D-like subgroups were excluded from further experiments (including the second gestation), as these groups did not model transient GDM.

\section{Confirmation of defective glucose handling by glucose tolerance tests}

We limited the use of prolonged fasting and OGTTs to validate the phenotype of glucose utilization in selected mouse groups or stages in order to minimize the risk of pregnancy stress or even loss of pregnant dams. At E16 of the first pregnancy, both GDM and isoIGT mice demonstrated a similar degree of severe postprandial glucose intolerance (Fig. 2C), while the effect of short-term HFD feeding on glucose intolerance was not significant.

Table 1 Experimental summary of dam outcomes.

\begin{tabular}{ll}
$\begin{array}{l}\text { Treatment } \\
\text { group }\end{array}$ & $\begin{array}{l}\text { Phenotypic } \\
\text { subgroup }\end{array}$ \\
\cline { 1 - 1 } LFD-Veh & - \\
LFD-STZ & - \\
HFD-Veh & - \\
HFD-STZ & T1D-like \\
HFD-STZ & GDM \\
HFD-STZ & T2D-like \\
HFD-STZ & isolGT \\
\hline
\end{tabular}

\begin{tabular}{ccc}
$\begin{array}{c}\text { Number of } \\
\text { animals }\end{array}$ & & HFD \\
\cline { 1 - 1 } 14 & & - \\
9 & \\
15 & & + \\
7 & + \\
24 & & + \\
6 & + \\
15 & & + \\
\hline
\end{tabular}

\begin{tabular}{l}
\hline STZ \\
\hline- \\
+ \\
- \\
+ \\
+ \\
+ \\
+ \\
\hline
\end{tabular}
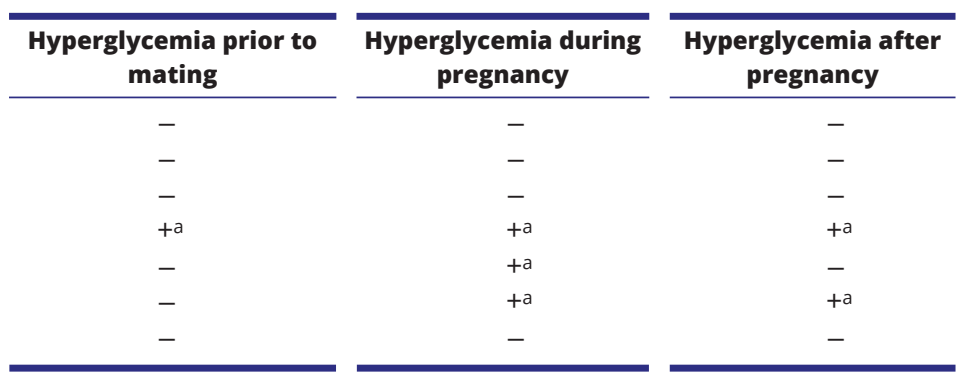

Experimental mice were grouped into four treatment groups: LFD-Veh, LFD-STZ, HFD-Veh, and HFD-STZ. Mice that received the HFD-STZ treatment were further categorized into four phenotypic subgroups based on glycemic outcome: T1D-like with hyperglycaemia prior to mating and hyperglycemia through pregnancy; T2D-like with hyperglycaemia only during pregnancy and after pregnancy; GDM with hyperglycaemia only during pregnancy; and isolGT normoglycemia prior to, during and after pregnancy. + represents applied treatment or random blood glucose $\geq 12 \mathrm{mM}$; - represents no treatment or random blood glucose $<12 \mathrm{mM}$.

a $P<0.05$, significance by two-way ANOVA.

GDM, a gestation-specific diabetic phenotype; T2D-like, a gestation and post-gestation diabetic phenotype; HFD, high-fat diet; isolGT, isolated impaired glucose tolerance; LFD, low-fat diet; RBG, random blood glucose; STZ, streptozotocin; T1D-like, a type 1 diabetes-like phenotype. 

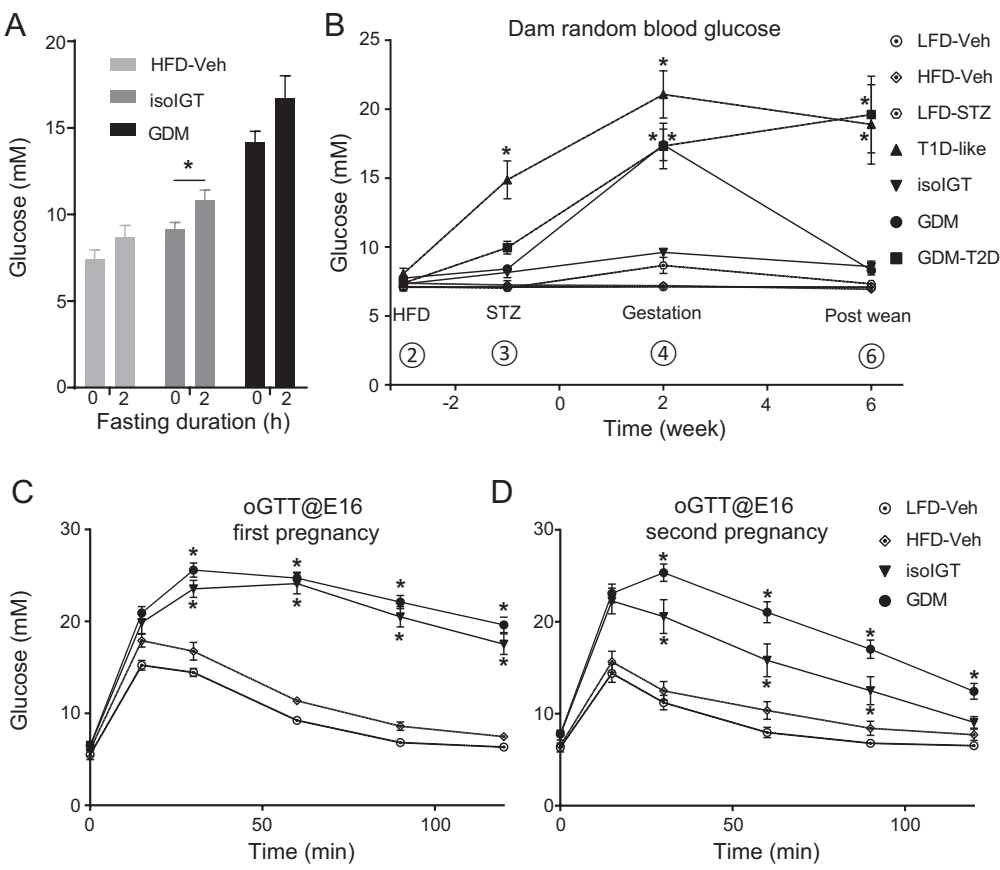
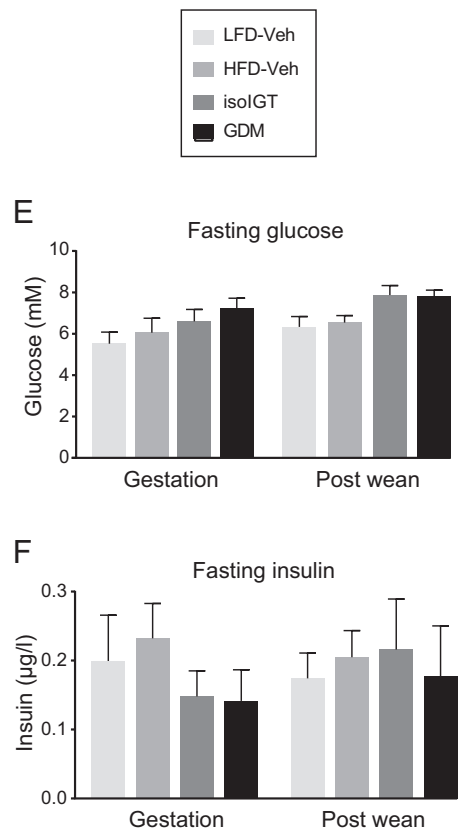

\section{Figure 2}

Transient hyperglycaemia in dams with gestational diabetes mellitus. (A) Random and 2-h fasting blood glucose levels of each group at E15. HFD-Veh $n=10$, isolGT $n=20$, GDM $n=12$. * $P<0.05$ with one-way ANOVA in group comparison. (B) Random blood glucose levels of each group during HFD exposure, following STZ exposure, during gestation and post weaning. Numbers in circles correspond to numbers in circles in Fig. $1 \mathrm{~A}$. LFD-Veh $n=14$, HFD-Veh $n=15$, LFD-STZ $n=9$, T1D-like $n=7$, isolGT $n=15$, GDM $n=24$, T2D-like $n=6$. (C) Oral glucose tolerance at E16 of first pregnancy on HFD (only results from pregnant animals are reported). (D) Oral glucose tolerance test at E16 of second pregnancy on chow diet (only results from pregnant animals are reported). (E) Fasting glucose levels at E16 and post weaning of first pregnancy. (F) Fasting insulin levels of indicated groups at E16 and post weaning of first pregnancy. For C, D, E and F, LFD-Veh $n=9$, HFD-Veh $n=12$, isolGT $n=11$, GDM $n=15$. * $p<0.05$, significance by two-way ANOVA. E, embryonic day; GDM, gestational diabetes mellitus; GDM-T2D, T2D-like; HFD, high-fat diet; isolGT, isolated impaired glucose tolerance; LFD, low-fat diet; OGTT, oral glucose tolerance test; STZ, streptozotocin; Veh, vehicle.

In their second pregnancy, both the GDM and isoIGT mice remained glucose intolerant despite the absence of an HFD challenge. Interestingly, the postprandial hyperglycemic phenotype was milder when compared to their first pregnancy, especially for the isoIGT animals and at the end of the 2-h test (Fig. 2D).

Fasting glucose levels (Fig. 2E) and insulin levels (Fig. 2F) during (gestation, E16) and after the first pregnancy (post wean, P30) were not significantly different between groups, although there was a visual trend of reduction for STZ-exposed mice in the first pregnancy.

These results confirm that the RBG measurement is a good surrogate marker to assess glucose tolerance and show that the GDM defined in the current model was associated with impaired glucose tolerance but normal fasting glycemia.

\section{Exacerbated risk of metabolic phenotype in the GDM offspring}

Although macrosomia could be a characteristic of GDM births, we chose not to weigh the offspring at birth,

(c) 2020 Society for Endocrinology Published by Bioscientifica Ltd. Printed in Great Britain so as not to disrupt maternal care and offspring survival. Male offspring of LFD-Veh, HFD-Veh, isoIGT and GDM dams were weaned to normal chow diet and followed for 16 weeks. Postnatal development under standard dietary conditions appeared normal with no difference in body weight and body fat content (Fig. 3A and B). There was also no significant difference in glucose tolerance between the groups when assessed between P98 and P112 (Fig. 3C).

We also examined a separate batch of offspring from the LFD-Veh and GDM dams following a challenge with HFD for 8 weeks starting at 8 weeks of age. At 16 weeks of age, they showed similar body weight (data not shown), but the GDM offspring displayed overt glucose intolerance (Fig. 3D), indicating their vulnerability to metabolic stress in adult life.

\section{Discussion}

We report here a two-hit protocol (HFD and STZ) to generate a GDM mouse model that displays transient and marked gestation-specific hyperglycemia, closely 
A
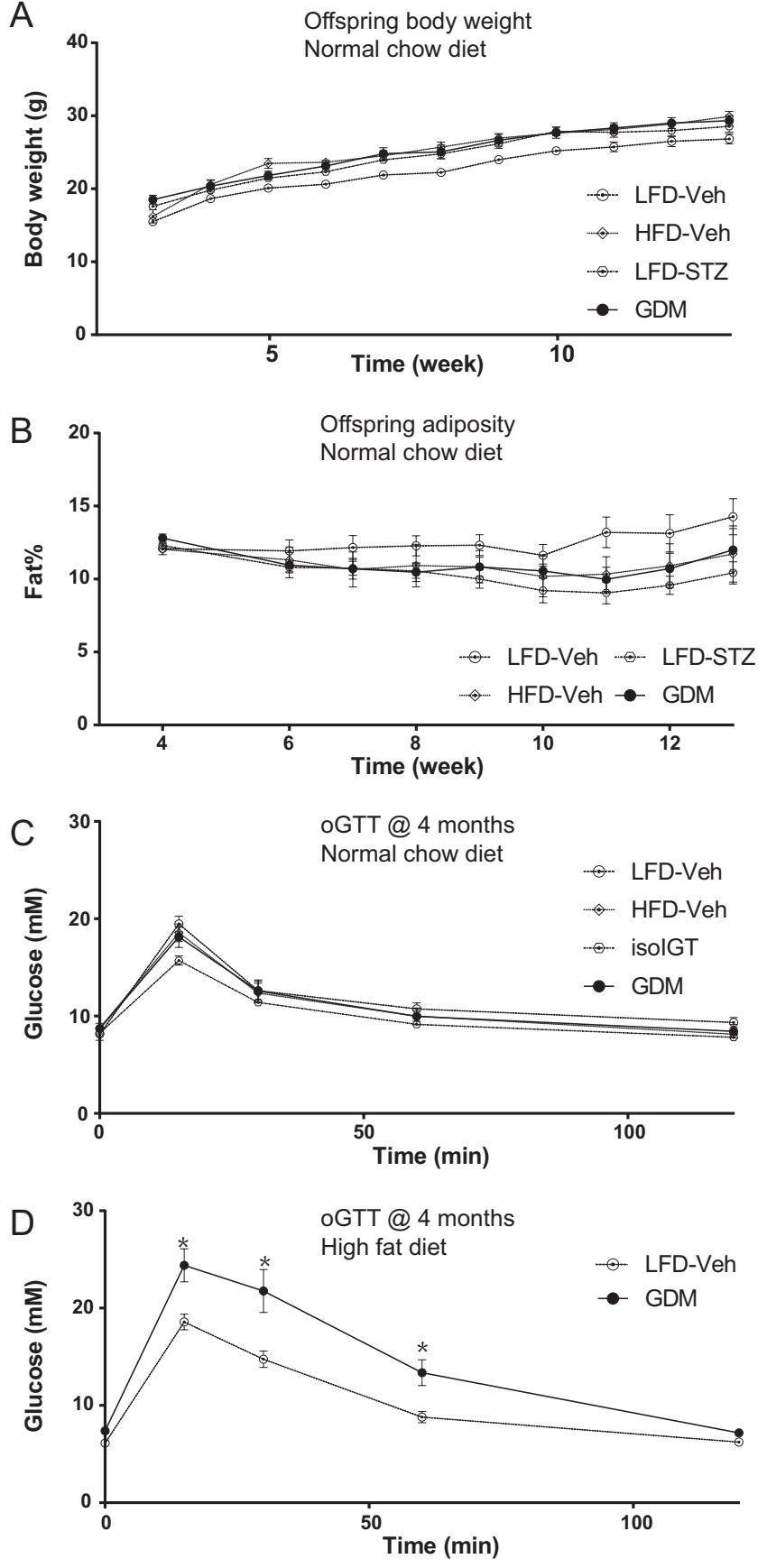

Figure 3

High-fat diet induces glucose intolerance in offspring of dams with gestational diabetes mellitus. (A) Body weight of offspring fed normal chow diet post weaning. (B) Adiposity (\% body fat) of offspring fed chow diet. (C) Oral glucose tolerance test at 4 months of age of offspring fed normal chow diet. (D) Oral glucose tolerance test at 4 months of age of offspring fed high-fat diet. LFD-Veh $n=6$, HFD-Veh $n=7$, isolGT $n=7$, GDM $n=10$. $* P<0.05$, significance by two-way ANOVA. HFD, high fat diet; GDM, gestational diabetes mellitus; LFD, low fat diet; isolGT, isolated impaired glucose tolerance; Veh, vehicle; STZ, streptozotocin. mimicking the development of GDM in human pregnancy. Our manipulations are relatively subtle compared to other preclinical GDM models and occur prior to pregnancy, minimizing environmental stressors to fetus/offspring. Our data suggest that such temporal and mild maternal GDM exposure increases vulnerability for metabolic disease in the offspring. Our protocol allows scalable generation of a large cohort of GDM dams and offspring with corresponding control groups, which can serve as an effective platform for both mechanistic studies and translational innovations.

Although mice and rats have been extensively used in metabolic studies, including glucose regulation and diabetes studies, the criteria for diabetes and GDM in rodents have not been clearly defined. In humans, repeated RBG measurements used to be a WHO criterion for GDM. However, this diagnostic standard was replaced with fasting OGTT in the 2013 guidelines, even though circadian blood glucose values of women with GDM (as determined by continuous glucose monitoring) continue to support RBG as a reliable criterion (Balsells et al. 2009). For a rodent GDM model generation and assessment, it is impractical to repeatedly fast and challenge the animals prior to mating and/or during gestation and lactation, as these manipulations inevitably affect pregnancy rates and normal progression of gestation, delivery and lactation. To minimize stress to the pregnant and/or lactating dams in our studies, we relied on morning RBG measurements without fasting and took measurements in two consecutive days to minimize potential measurement inconsistency when needed. In fact, we noticed that morning RBG was a more sensitive measurement than 2-h fasting glucose, overnight fasting glucose or OGTT. The 2-h fasting during gestation resulted in fluctuating and overall elevated readings, especially for diabetic dams; this could be attributed to animal stress in response to the additional handling. However, the RBG readings could be obtained with minimal handling by trained personnel, thus ensuring more reproducible results. The overnight fasting glucose levels were not different across groups, indicating that the fasting during gestation were severe enough to mask the difference between control and diabetic groups. Meanwhile, OGTT failed to differentiate isoIGT and GDM groups regardless of their distinct RBG profile. Thus, we believe that, in a transient diabetes model like ours, the RBG measurement remains a valuable approach to reflect the actual physiology of these dysregulated phenotypes, 
at least in rodent gestation studies. These questions could be further explored with the aid of continuous glucose monitoring techniques; however, these are as yet untested during rodent gestation.

In the literature, several glucose levels, ranging from 10 to $15 \mathrm{mM}$, have been used to define hyperglycemia and diabetes in mice (Tutino et al. 2014). Although there is currently no consensus on the diagnosis value for diabetes in animal studies, we chose $12 \mathrm{mM} \mathrm{RBG}$ as the diagnostic threshold for the following reasons: (1) it is the upper limit of mouse post-prandial blood glucose levels detected in wild-type mice (based on data from years of assessment in our laboratory) and (2) we did not observe any reading above this line in the control groups, even under stressful conditions, such as after 2-h fasting. Therefore, it's unlikely to be confused with fluctuations due to normal activities or compromised conditions. We also confirmed by using OGTT that animals with RBG above $12 \mathrm{mM}$ display unequivocal glucose intolerance. Standardization of these measurements is lacking. However, from our data, we conclude that an RBG level of $12 \mathrm{mM}$ can efficiently identify glucose-intolerant mice, and thus, propose it as a screening criterion for GDM across preclinical studies in C57/B16 mice.

We aimed to generate a mouse model that resembles clinically relevant pathogenesis. Human GDM is, in essence, a transient type of diabetes, which is the result of failed beta-cell compensation of peripheral insulin resistance during late stage pregnancy. HFD and STZ are frequently used to induce obesity-related insulin insensitivity and loss of beta-cells, respectively. However, the majority of women with GDM are not obese (Coustan et al. 2010, Lapolla et al. 2011), and the commonly used dosages of STZ cause dramatic loss of beta-cells and consequently hyperglycemia, similar to type 1 diabetes prior to pregnancy. Furthermore, STZ given during gestation to limit the hyperglycemia to late pregnancy has been reported to have harmful effects on fetal development (Giavini et al. 1986, Kalter 1996, Turhan et al. 2018). To circumvent these pitfalls, we decided to develop a combined treatment protocol that includes a short HFD feeding, which would not induce drastic body weight gain (Fig. 1B), with a low dose of STZ, to undermine beta cell compensation to insulin resistance during late gestation while preserving, as much as possible, normal insulin levels, similar to human GDM. Moreover, STZ administration was completed prior to mating, with animals displaying normoglycemia prior to gestation and (in a considerable number of treated animals) full recovery to normoglycemia after delivery.
The normal fasting glucose and insulin levels observed during gestation and after weaning can be translated to human diagnostic parameters and indicate that our STZ treatment is relatively mild compared to other preclinical GDM models that rely on STZ exposure (Wu \& Huan 2008, Pasek \& Gannon 2013). It is also worth noting that our two-hit protocol requires both HFD and STZ treatments, as individual treatment with either HFD or STZ was insufficient to induce GDM.

Any model that adequately captures the heterogeneity in glycemic outcomes, mimicking the human GDM phenotype, will require large numbers of animals. The GDM rate using this approach was relatively high; our conversion rate of approximately $50 \%$ is realistic and acceptable given the heterogeneity of responses to the individual HFD or STZ treatment. Moreover, we observed distinct subgroups according to RBG levels in mice treated with HFD-STZ, indicating differing responses to the twohit procedure. Some mice were more vulnerable to the treatment procedures, and thus developed persistent diabetes. While some other mice were more resistant to the challenges, and remained euglycemic. Considering that the majority of the mice were the GDM mice, suggesting that we have optimized the environmental manipulations under the heterogenous influence on the development of GDM. Fine-tuning of the parameters may alter the ratio of subgroups, and such experimental outcome is comparable to the various grades of GDM as observed in humans. Thus, our model provides a wide range of possible subgroups for various study design needs.

In humans, GDM has been reported to be associated with a broad range of adverse health outcomes including, increasingly, fetal and postnatal growth. In our model, GDM offspring maintained on normal chow diet after weaning showed normal body weight gain, body fat content and glucose tolerance at 4 months of age. However, when fed with HFD for 2 months at the start of adolescence, the GDM offspring developed overt glucose intolerance, consistent with higher sensitivity to environmental and metabolic stress following prenatal exposure of adverse metabolic conditions. This indicates the possibility of further and more severe adverse consequence for these offspring in later life, such as the development of obesity, T2D or symptoms associated with metabolic syndrome. We believe that our current model shows clinical relevance and translation value to human GDM and GDM infants/children, as it captures the essential characteristics of the pathogenesis of GDM and heightened sensitivity of GDM offspring to metabolic stress in adult life. The improved glucose tolerance evident 
in the second pregnancy (as compared to the glucose intolerance in the first pregnancy) occurred without active exposure to HFD; it is possible that continued exposure to the HFD would have led to worse outcomes indicating the possibility of active nutritional interventions for the mother to prevent GDM in subsequent pregnancies. Mechanistic and discovery studies based on the current GDM model may lead to the development of diagnostic markers and treatment strategies, especially during early life, for GDM offspring. Moreover, the current model may allow examination of the efficacy of preventative or therapeutic interventions in the first and subsequent pregnancy, as well as in the offspring.

\section{Declaration of interest}

The authors declare that there is no conflict of interest that could be perceived as prejudicing the impartiality of the research reported.

\section{Funding}

This work was supported by A*STAR Biomedical Research Council (W H) and Danone Nutricia Research. E v d B, S S, Y L, and L H are employees of Danone Nutricia Research.

\section{References}

Balsells M, Garcia-Patterson A, Gich I \& Corcoy R 2009 Maternal and fetal outcome in women with type 2 versus type 1 diabetes mellitus: a systematic review and metaanalysis. Journal of Clinical Endocrinology and Metabolism 94 4284-4291. (https://doi.org/10.1210/jc.20091231)

Bimson BE, Rosenn BM, Morris SA, Sasso EB, Schwartz RA \& Brustman LE 2017 Current trends in the diagnosis and management of gestational diabetes mellitus in the United States. Journal of Maternal-Fetal and Neonatal Medicine 30 2607-2612. (https://doi.org/10.1080/14767058. 2016.1257603)

Chong YS, Cai S, Lin H, Soh SE, Lee YS, Leow MK, Chan YH, Chen L, Holbrook JD, Tan KH, et al. 2014 Ethnic differences translate to inadequacy of high-risk screening for gestational diabetes mellitus in an Asian population: a cohort study. BMC Pregnancy and Childbirth 14 345. (https://doi.org/10.1186/1471-2393-14-345)

Coustan DR, Lowe LP, Metzger BE, Dyer AR \& International Association of Diabetes and Pregnancy Study Groups 2010 The Hyperglycemia and Adverse Pregnancy Outcome (HAPO) study: paving the way for new diagnostic criteria for gestational diabetes mellitus. American Journal of Obstetrics and Gynecology 202 654.e1-654.e6. (https://doi. org/10.1016/j.ajog.2010.04.006)

Ehrlich SF, Hedderson MM, Feng J, Davenport ER, Gunderson EP \& Ferrara A 2011 Change in body mass index between pregnancies and the risk of gestational diabetes in a second pregnancy. Obstetrics and Gynecology 117 1323-1330. (https://doi.org/10.1097/ AOG.0b013e31821aa358)

Eleazu CO, Eleazu KC, Chukwuma S \& Essien UN 2013 Review of the mechanism of cell death resulting from streptozotocin challenge in experimental animals, its practical use and potential risk to humans. Journal of Diabetes and Metabolic Disorders 12 60. (https://doi. org/10.1186/2251-6581-12-60)

Farahvar S, Walfisch A \& Sheiner E 2019 Gestational diabetes risk factors and long-term consequences for both mother and offspring: a literature review. Expert Review of Endocrinology and Metabolism 14 63-74. (https://doi.org/10.1080/17446651.2018.1476135)

Farrar D, Simmonds M, Bryant M, Sheldon TA, Tuffnell D, Golder S \& Lawlor DA 2017 Treatments for gestational diabetes: a systematic review and meta-analysis. BMJ Open 7 e015557. (https://doi. org/10.1136/bmjopen-2016-015557)

Getahun D, Fassett MJ \& Jacobsen SJ 2010 Gestational diabetes: risk of recurrence in subsequent pregnancies. American Journal of Obstetrics and Gynecology 203 467.e1-467.e6. (https://doi.org/10.1016/j. ajog.2010.05.032)

Giavini E, Broccia ML, Prati M, Roversi GD \& Vismara C 1986 Effects of streptozotocin-induced diabetes on fetal development of the rat. Teratology 34 81-88. (https://doi.org/10.1002/tera.1420340111)

Gustavsson N, Wei SH, Hoang DN, Lao Y, Zhang Q, Radda GK, Rorsman P, Sudhof TC \& Han W 2009 Synaptotagmin-7 is a principal $\mathrm{Ca} 2+$ sensor for $\mathrm{Ca} 2+$-induced glucagon exocytosis in pancreas. Journal of Physiology 587 1169-1178. (https://doi.org/10.1113/ jphysiol.2008.168005)

Heydemann A An overview of murine high fat diet as a model for type 2 diabetes mellitus. Journal of Diabetes Research 2016 2902351. (https:// doi.org/10.1155/2016/2902351)

Kaaja R \& Ronnemaa T 2008 Gestational diabetes: pathogenesis and consequences to mother and offspring. Review of Diabetic Studies $\mathbf{5}$ 194-202. (https://doi.org/10.1900/RDS.2008.5.194)

Kalter H 1996 Reproductive toxicology in animals with induced and spontaneous diabetes. Reproductive Toxicology 10 417-438. (https:// doi.org/10.1016/s0890-6238(96)00129-3)

Kampmann U, Madsen LR, Skajaa GO, Iversen DS, Moeller N \& Ovesen P 2015 Gestational diabetes: a clinical update. World Journal of Diabetes 6 1065-1072. (https://doi.org/10.4239/wjd.v6.i8.1065)

Lapolla A, Dalfra MG, Ragazzi E, De Cata AP \& Fedele D 2011 New International Association of the Diabetes and Pregnancy Study Groups (IADPSG) recommendations for diagnosing gestational diabetes compared with former criteria: a retrospective study on pregnancy outcome. Diabetic Medicine 28 1074-1077. (https://doi. org/10.1111/j.1464-5491.2011.03351.x)

Li H, Wei S, Cheng K, Gounko NV, Ericksen RE, Xu A, Hong W \& Han W 2014 BIG3 inhibits insulin granule biogenesis and insulin secretion. EMBO Reports 15 714-722. (https://doi.org/10.1002/ embr.201338181)

Logan KM, Gale C, Hyde MJ, Santhakumaran S \& Modi N 2017 Diabetes in pregnancy and infant adiposity: systematic review and meta-analysis. Archives of Disease in Childhood: Fetal and Neonatal Edition 102 F65-F72. (https://doi.org/10.1136/ archdischild-2015-309750)

Lowe Jr WL, Scholtens DM, Kuang A, Linder B, Lawrence JM, Lebenthal Y, McCance D, Hamilton J, Nodzenski M, Talbot O, et al. 2019 Hyperglycemia and adverse pregnancy outcome follow-up study (HAPO FUS): maternal gestational diabetes mellitus and childhood glucose metabolism. Diabetes Care 42 372-380. (https://doi. org/10.2337/dc18-1646)

Pasek RC \& Gannon M 2013 Advancements and challenges in generating accurate animal models of gestational diabetes mellitus. American Journal of Physiology: Endocrinology and Metabolism 305 E1327-E1338. (https://doi.org/10.1152/ajpendo.00425.2013)

Shoelson SE, Herrero L \& Naaz A 2007 Obesity, inflammation, and insulin resistance. Gastroenterology 132 2169-2180. (https://doi.org/10.1053/j. gastro.2007.03.059)

Turhan U, Yilmaz E, Gul M, Melekoglu R, Turkoz Y, Ozyalin F, Parlakpinar H \& Simsek Y 2018 Investigation of the effect of gestational diabetes on fetal cardiac tissue in streptozotocin induced https://joe.bioscientifica.com https://doi.org/10.1530/JOE-19-0516 (c) 2020 Society for Endocrinology Published by Bioscientifica Ltd. Printed in Great Britain 
in rats. Acta Cirurgica Brasileira 33 306-313. (https://doi.org/10.1590/ s0102-865020180040000002)

Tutino GE, Tam WH, Yang X, Chan JC, Lao TT \& Ma RC 2014 Diabetes and pregnancy: perspectives from Asia. Diabetic Medicine 31 302-318. (https://doi.org/10.1111/dme.12396)

Winzell MS \& Ahren B 2004 The high-fat diet-fed mouse: a model for studying mechanisms and treatment of impaired glucose tolerance and type 2 diabetes. Diabetes 53 (Supplement 3) S215-S219. (https:// doi.org/10.2337/diabetes.53.suppl_3.s215)

Wu KK \& Huan Y 2008 Streptozotocin-induced diabetic models in mice and rats. Current Protocols in Pharmacology Chapter 5 Unit 5.47. (https://doi.org/10.1002/0471141755.ph0547s40)
Yamamoto JM, Kellett JE, Balsells M, Garcia-Patterson A, Hadar E, Sola I, Gich I, van der Beek EM, Castaneda-Gutierrez E, Heinonen S, et al. 2018 Gestational diabetes mellitus and diet: a systematic review and meta-analysis of randomized controlled trials examining the impact of modified dietary interventions on maternal glucose control and neonatal birth weight. Diabetes Care 41 1346-1361. (https://doi. org/10.2337/dc18-0102)

Yew TW, Khoo CM, Thai AC, Kale AS, Yong EL \& Tai ES 2014 The prevalence of gestational diabetes mellitus among Asian females is lower using the new 2013 World Health Organization diagnostic criteria. Endocrine Practice 20 1064-1069. (https://doi.org/10.4158/ EP14028.OR)

Received in final form 11 November 2019

Accepted 23 December 2019

Accepted Manuscript published online 7 January 2020
(C) 2020 Society for Endocrinology Published by Bioscientifica Ltd. 